\title{
Un recuento de argumentos para incluir a los artrópodos terrestres en las prácticas de evaluación ambiental
}

\author{
Alfredo E. Giraldo Mendoza \\ Laboratorio de Ecología de Procesos. Universidad Nacional Agraria La Molina, Lima, Perú.
}

\begin{abstract}
[Palabras clave: hipótesis de la discontinuidad textural, interacciones artrópodo-planta y artrópodo-vertebrado, propiedades y procesos ecosistémicos]
\end{abstract}

[Keywords: textural discontinuity hypothesis, arthropod-plant and arthropod-vertebrate interactions, ecosystem properties and process]

El contexto actual de acelerada modificación humana de los ecosistemas terrestres ha generado una demanda creciente de prácticas de evaluación ambiental que sean viables en términos económicos, relativamente rápidas y sustentadas en el conocimiento científico disponible. Este tipo de evaluaciones requiere una interacción social intensa, en la cual los científicos deberían aportar información útil para las decisiones que competen a los gestores de ecosistemas (Turnhout et al. 2007). Una respuesta de la ciencia frente a estas exigencias es el uso de indicadores, es decir, parámetros o variables biológicas, físicas, químicas y sociales que permiten detectar si el ecosistema se ha apartado de aquellos estados que son biofísicamente posibles y compatibles con las necesidades de una sociedad caracterizada por una pluralidad de valores contradictorios (Noss 1990).

En los ecosistemas terrestres, los artrópodos han sido propuestos como potenciales indicadores biológicos a través de investigaciones presentadas en un lapso de tres décadas (Andersen 1999). La propuesta tiene concordancia con los atributos biológicos de los artrópodos en la mayoría de los ecosistemas terrestres: número elevado de individuos, biomasa combinada superior a la alcanzada por los vertebrados, gran cantidad de especies, y la variedad de roles que cumplen en las redes tróficas (Cardoso et al. 2011). Asimismo, la evidencia empírica señala que pueden ser útiles para describir patrones de biodiversidad y además sensibles a las perturbaciones antropogénicas (Maleque et al. 2009).

En contraste con los atributos biológicos que se reconocen para los artrópodos terrestres, las reacciones humanas más frecuentes hacia ellos son el desinterés, el miedo y la aversión (Melic 2003). Esta percepción negativa desalienta la inversión de recursos financieros y humanos para el conocimiento y la conservación de artrópodos, perpetuando los vacíos de información existentes y reduciendo al mínimo su inclusión en los planes de conservación (Cardoso et al. 2011).

A continuación se exponen cuatro argumentos para incluir a los artrópodos terrestres en las prácticas de evaluación ambiental; para ello se recurre a la evidencia empírica obtenida por investigadores en distintos ecosistemas del mundo. Estos argumentos pueden ser útiles para facilitar la interacción entre gestores de ecosistemas y científicos en la fase de planeamiento de una evaluación ambiental. La mayor parte de la evidencia presentada a favor de estos argumentos se refiere al nivel de organización comunitario, con alusiones a los niveles de organización inmediatamente superior (paisaje) e inferior (población). La evaluación ambiental se define aquí como la valoración de los impactos provocados por las actividades antrópicas, que aportan elementos de juicio para la toma de decisiones políticas. Por lo tanto, se trata de una definición amplia que no se refiere sólo a procedimientos administrativos que sirven de requisito o compromiso legal para algunas actividades humanas.

Editora asociada: Adriana Salvo 


\section{RECUENTO DE ARGUMENTOS}

\section{Los artrópodos discriminan las pequeñas discontinuidades del paisaje que son desapercibidas por los vertebrados}

La hipótesis de la discontinuidad textural postula que los animales demuestran la existencia de una estructura jerárquica y de una textura discontinua en el paisaje que habitan; mostrando a su vez distribuciones discontinuas en sus tamaños, escalas de búsqueda y opciones de comportamiento (Holling 1992). A partir de este enunciado se puede intuir que los artrópodos y los vertebrados interactúan con el paisaje en diferentes escalas espaciales debido a sus diferencias de tamaño corporal. Para ilustrar esta afirmación, los datos de masa corporal

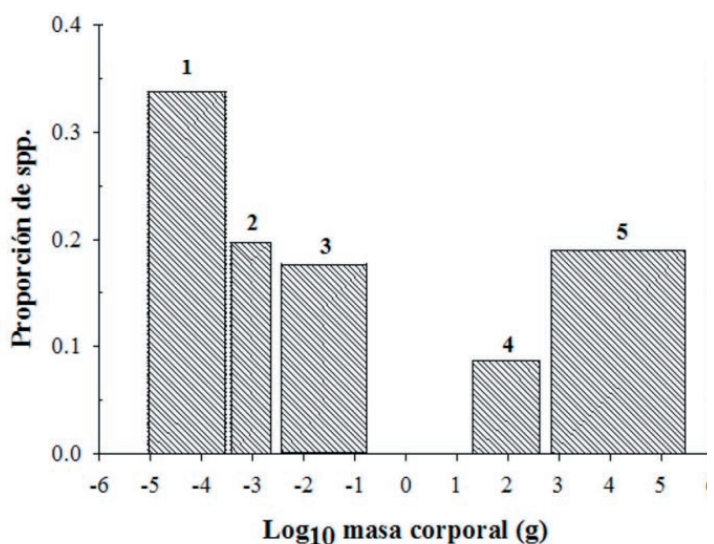

a) Clases obtenidas con un análisis de cluster univariado (método de Ward).

Clumps obtained with a univariate cluster analysis (Ward's method).

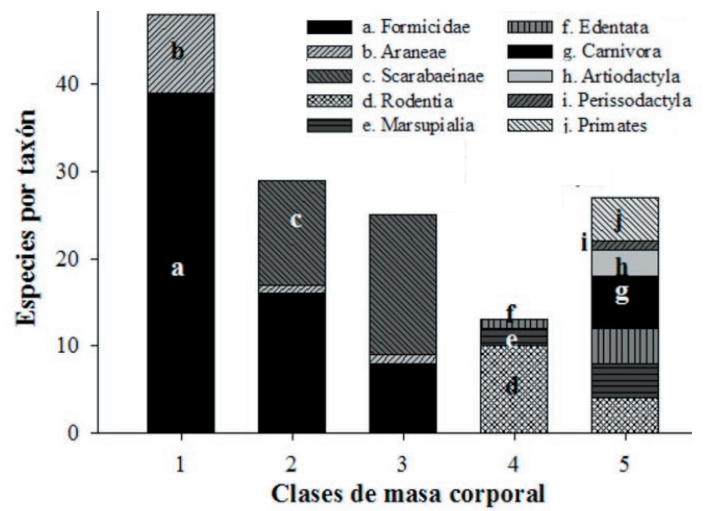

b) Composición de taxones en cada clase de masa corporal. Taxonomic composition in each body mass clump.

Figura 1. Clases de masa corporal para 142 especies de artrópodos y mamíferos en la isla Barro Colorado.

Figure 1. Body mass clumps for 142 species of arthropods and mammals in Barro Colorado island. de un conjunto de especies de la isla Barro Colorado (Panamá) fueron agrupados en clases por medio de un análisis "cluster" univariado con distancias euclídeas y el método de unión de Ward (Wardwell et al. 2008). Las cinco clases de masa corporal resultantes (Figura 1a y b) muestran que los artrópodos y los mamíferos presentan rangos de masa corporal separados, cada uno de ellos con una clase de masa corporal más frecuente bien definida. Esta imagen obtenida a partir de una fracción de la fauna de Barro Colorado tiene semejanzas notables con la distribución de masas corporales de todas las especies de animales que habitan en la Isla Marion (Gaston et al. 2001).

La humanidad ha transformado la biosfera de una manera drástica; la ha convertido en un mosaico de fragmentos de ecosistemas naturales y antropogénicos, con un aumento progresivo de la superficie de estos últimos. Hasta cierto punto, este es un contexto favorable para el uso de artrópodos en evaluaciones de biodiversidad dado que pueden brindar información acerca de los cambios ecológicos que se producen en escalas espaciales pequeñas. Una serie de investigaciones muestran que los artrópodos pueden aportar información útil para la gestión de escenarios naturales de reducida extensión, como ha sido evidenciado en cuevas (Ferreira \& Horta 2001). Esta capacidad para generar información se aplica también para ambientes "insulares" resultado de la transformación antropogénica del paisaje (e.g., jardines y fragmentos de vegetación nativa inmersos en matrices urbanas) (Bolger et al. 2000; Smith et al. 2006).

Un aspecto importante en la gestión de ecosistemas es comprobar si la configuración del paisaje es compatible con el mantenimiento de la biodiversidad, puesto que los bordes entre parches pueden servir como vías o barreras para la dispersión de organismos según sus características de vegetación y suelo. Este fenómeno ha sido documentado para artrópodos terrestres en bordes de carreteras (Haskell 2000) y en bordes de cultivos (Thomas \& Marshall 1999).

Los artrópodos se relacionan estrechamente con plantas y vertebrados que tienen un elevado valor de conservación

Una posibilidad más para incluir a los artrópodos terrestres en las prácticas de 
evaluación ambiental es vincularlos con las plantas y los vertebrados que suelen ser objeto de la gestión de ecosistemas. Lejos de ser artificios, los vínculos entre estos organismos son interacciones biológicas que se han establecido a través de millones de años, como lo sugieren las evidencias del registro fósil. Por ejemplo, hojas fósiles de angiospermas con perforaciones que se atribuyen a orugas minadoras de las familias Gracillariidae y Nepticulidae del Cretácico Medio (Labandeira et al. 1994). En cuanto a las relaciones entre artrópodos y vertebrados, se puede citar el hallazgo de coprolitos de dinosaurios del Cretácico Tardío que presentan galerías atribuidas a escarabajos de la subfamilia Scarabaeinae (Chin \& Gill 1996).

Alrededor del mundo, la gestión de ecosistemas tiene como uno de sus problemas más apremiantes la introducción accidental o intencionada de especies vegetales exóticas que han reemplazado de forma progresiva a las especies vegetales nativas. Más allá de los evidentes cambios en la flora, suelen producirse cambios en la abundancia, la diversidad y la composición de artrópodos. Estos cambios conciernen principalmente a los fitófagos y antófilos, a los predadores y parasitoides de los fitófagos, y también a otros que utilizan las estructuras vegetales como refugio o sitio de apareamiento. Se han obtenido evidencias de estos procesos en humedales costeros (Gratton \& Denno 2005) y matorrales (Harris et al. 2004).

Los vertebrados ocupan un lugar protagónico en la gestión de ecosistemas, al punto que gran parte de las áreas naturales protegidas y de los instrumentos legales de protección ambiental están destinados a mantener la viabilidad de sus poblaciones. Puesto que entre los vertebrados útiles para las actividades de caza, pesca y turismo se encuentran especies insectívoras, los artrópodos deberían ser gestionados como un recurso que sustenta en última instancia la viabilidad de dichas actividades humanas (Losey \& Vaughan 2006). La preocupación por mantener una biomasa de artrópodos que sirva de sustento para los vertebrados insectívoros se ve reflejada en algunas investigaciones referidas a prácticas de manejo y restauración forestal (Chan et al. 2007). El ciclo biológico de algunos insectos depende de la disponibilidad de cadáveres y excrementos de vertebrados, de modo que su abundancia y diversidad son comprometidas cuando las poblaciones de vertebrados son mermadas a causa de la caza y la transformación del paisaje (Nichols et al. 2009).

En otras circunstancias, la actividad de los vertebrados puede tener consecuencias para la abundancia y diversidad de artrópodos. Algunos roedores pueden transformar los microhábitats de los artrópodos a través de cambios físicos en el suelo, como ha sido documentado para los perritos de las praderas (Bangert \& Slobodchikoff 2006). Asimismo, la introducción de algunos vertebrados en islas ha disminuido la biodiversidad de artrópodos nativos a través de la compactación del suelo (Vtorov 1993) o la depredación (Palmer \& Pons 1996).

Los artrópodos pueden brindar información acerca de propiedades y procesos ecosistémicos

\section{dificilmente cuantificables}

La complejidad inherente a los ecosistemas ha sido descrita por la Ecología mediante una serie de conceptos tales como estabilidad, integridad, madurez, resiliencia, salud, sostenibilidad, etc. (Margalef 1991). Aunque muchos de estos conceptos son auténticas "cajas negras" difíciles de cuantificar e incluso de definir, son necesarios para implementar la gestión de ecosistemas con base en la teoría ecológica. En este punto, la evaluación de artrópodos terrestres podría ser útil puesto que sus patrones de abundancia y diversidad no dependen de variables ambientales aisladas, sino de un intrincado conjunto de variables de suelo, topografía y vegetación (Ribera \& Foster 1997).

La sostenibilidad ha sido sugerida como el concepto global para regular las prácticas de manejo en los agroecosistemas, de manera que haya un balance entre la productividad agrícola y el mantenimiento de los servicios ecosistémicos. En este contexto, son tres los servicios ecosistémicos que involucran a distintos grupos de artrópodos terrestres: depredadores y parasitoides en el control de plagas, detritívoros en el reciclaje de nutrientes en el suelo einsectos antófilos en la polinización de cultivos. Por lo tanto, la sostenibilidad de un agroecosistema puede ser medida a través de su biodiversidad de artrópodos benéficos, y en tal sentido se ha desarrollado un volumen importante de investigación sobre los efectos que pueden tener el labrado del suelo, el uso de fertilizantes, la configuración de cercos vivos, la rotación de cultivos y el uso de 
pesticidas en la abundancia y diversidad de estos artrópodos (Paoletti et al. 2007).

Los bosques son ecosistemas maduros que albergan gran cantidad de diversidad biótica, sustentada principalmente en la arquitectura compleja de los árboles, la acumulación de hojarasca en el suelo, y la madera en distintos estados de descomposición. En bosques manejados, las prácticas de tala provocan cambios en la complejidad estructural que pueden desembocar en distintos estados alternativos que promueven o reducen la biodiversidad. En bosques severamente talados, las prácticas de restauración promueven las condiciones iniciales para un lento proceso de resiliencia que debería restituir los elementos y funciones de un bosque primario. Ambos son procesos estudiados en reiteradas oportunidades a través de las comunidades de artrópodos y en base a ellas, varios taxones han sido propuestos como potenciales indicadores (Maleque et al. 2009).

En distintos ecosistemas se ha propuesto el concepto de integridad biótica, como aquel estado libre o mínimamente afectado por la intervención humana que puede servir como punto de referencia para medir la intensidad y dirección de las perturbaciones antropogénicas. Las comunidades de artrópodos al estar compuestas por una variedad de taxones, grupos funcionales y especies con nichos bien diferenciados, aportan gran cantidad de variables o métricas que pueden integrarse en los llamados índices de integridad biótica. La elaboración de índices de integridad biótica con datos de artrópodos se ha realizado en matorrales de Norteamérica (Karr \& Kimberling 2003) y en las islas Azores (Cardoso et al. 2007).

Los artrópodos pueden ser evaluados a un bajo costo sin comprometer la información obtenida de ellos

Las evaluaciones ambientales son actividades humanas que requieren mano de obra, materiales e infraestructura, las cuales, en conjunto, son una inversión de tiempo y dinero. Si se aplica este razonamiento al componente biótico de la evaluación ambiental, se debería buscar que sean costoefectivas, es decir, que haya un adecuado balance entre la información que se obtiene de los taxones evaluados y la inversión realizada. Con respecto a los artrópodos terrestres, Andersen (1999) señaló que éstos serán fácilmente adoptados en las prácticas de manejo de ecosistemas cuando además de demostrar que son indicadores biológicos fiables, se logre elaborar protocolos prácticos para los trabajos de campo y de laboratorio.

En una primera impresión, el tamaño reducido y la riqueza elevada convierten a los artrópodos terrestres en organismos particularmente costosos de evaluar en comparación con las plantas y con los vertebrados. Sin embargo, tres investigaciones realizadas en bosques tropicales de Camerún (Lawton et al. 1998), en matorrales de Australia (Bisevac \& Majer 2002) y en bosques tropicales de Brasil (Gardner et al. 2008) sugieren que tal impresión no siempre es cierta. Según se observa en la Figura 2, varios taxones de artrópodos presentaron altos valores de riqueza (diversidad $\alpha$ ) y de recambio de especies (diversidad $\beta$ ), con una inversión de tiempo moderada para completar el trabajo de campo y laboratorio. Este fue el caso de los Coleoptera de dosel y voladores (Figura 2a), Coleoptera y Formicidae (Figura 2b), y Arachnida, Nymphalidae y Scarabaeinae (Figura 2c). En consecuencia, pese a los diferentes métodos de captura, ecosistemas y grados de perturbación involucrados, la mayor efectividad de costo correspondió en todos los casos a una serie de taxones de artrópodos terrestres, al mismonivel alcanzado por aves y plantas. También es cierto que la efectividad de costo no fue favorable para otros taxones de artrópodos terrestres, ya sea por su contribución limitada a la diversidad $\alpha$ (Chilopoda, Euglossini, Isopoda y Oestroidea) o porque su tamaño reducido incrementa de modo considerable el tiempo invertido en la revisión de los especímenes (Collembola, Drosophilinae e Isoptera). Entre los taxones con menor efectividad de costo encontramos también a los nemátodos (los cuales requieren tiempos prolongados para examinar los especímenes en laboratorio) y a los anfibios, mamíferos y reptiles (cuyas contribuciones a la diversidad ( $\alpha$ y $\beta$ ) fueron menores a las observadas para otros taxones).

En ocasiones, las evaluaciones de artrópodos terrestres pueden ganar costo-efectividad si se aprovechan los ejemplares colectados incidentalmente con métodos de captura diseñados para la colecta de vertebrados. Las trampas de caída para colectar herpetofauna son baldes vacíos enterrados al ras del suelo, con un volumen de entre 20 a $35 \mathrm{~L}$, 
que también han resultado útiles para el muestreo de comunidades de artrópodos epigeos en distintos ecosistemas, incluyendo Arachnida, Curculionidae, Formicidae y Orthoptera (Andersen et al. 2002; Fabricius et

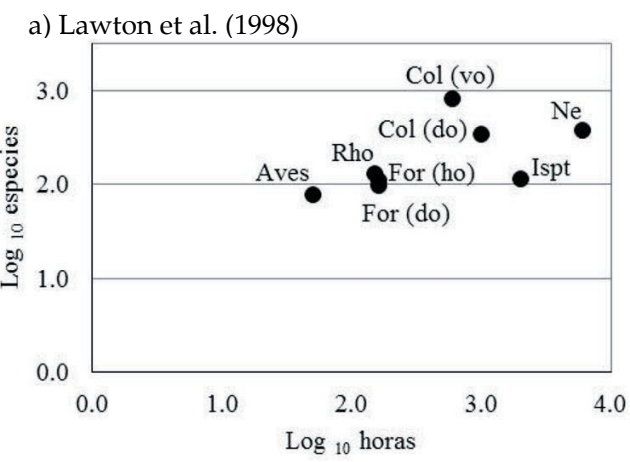

b) Bisevac and Majer (2002)

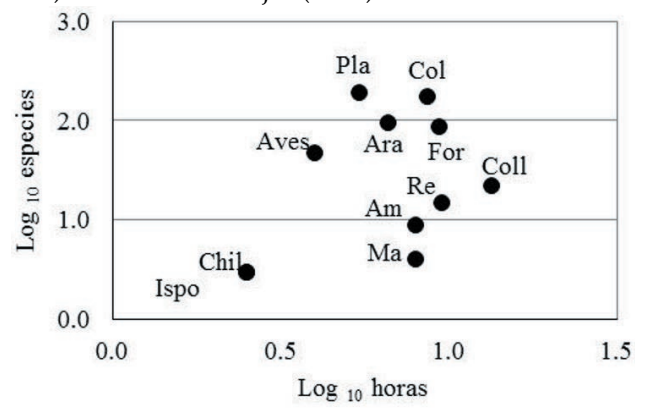

c) Gardner et al. (2008)

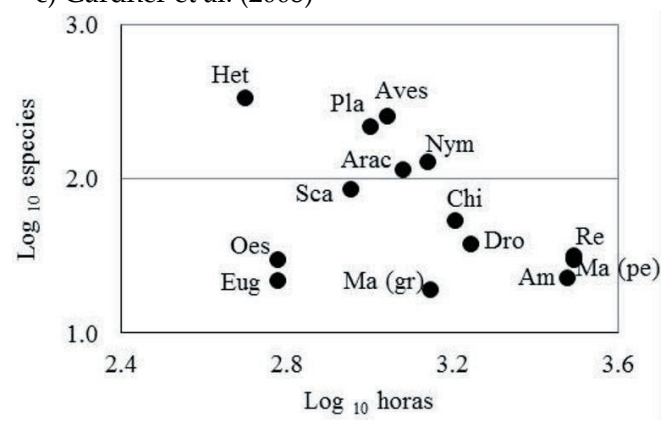

al. 2003). Cabe destacar que Andersen et al. (2002) consiguieron evaluar la respuesta de las comunidades de hormigas a la polución por dióxido de azufre a partir de colectas incidentales, reproduciendo los patrones de
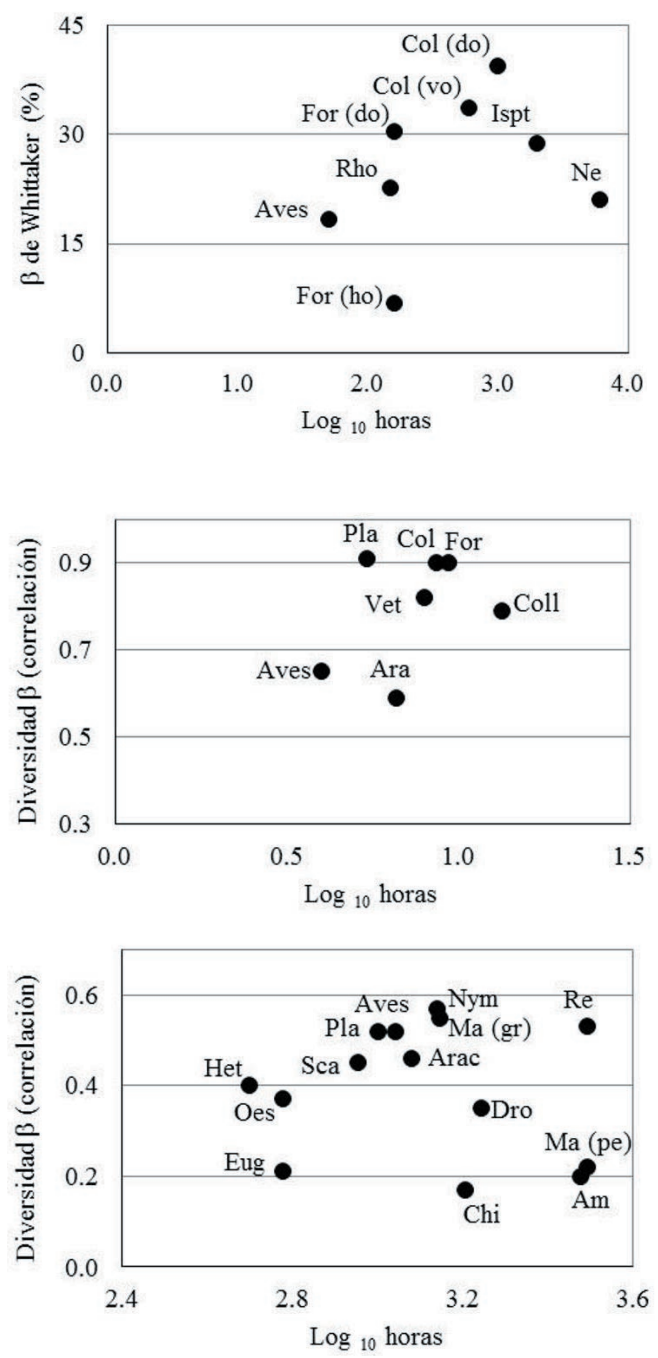

Figura 2. Efectividad de costo en evaluaciones biológicas de distintos taxones de invertebrados, plantas y vertebrados.

Figure 2. Cost-effectiveness in bio-assessments of several taxons of invertebrates, plants and vertebrates.

Abreviaturas: $\mathrm{Am}=$ Amphibia, $\mathrm{Ara}=$ Araneae, $\mathrm{Arac}=$ Arachnida $($ Amblypygi, Araneae, Opiliones, Scorpiones $\mathrm{y}$ Uropygi), $\mathrm{Chi}=$ Chiroptera, Chil $=$ Chilopoda, $\mathrm{Col}(\mathrm{do})=$ Coleoptera del dosel, $\mathrm{Col}(\mathrm{vo})=$ Coleoptera voladores, $\mathrm{Col}$ $=$ Coleoptera, Coll $=$ Collembola, Dro $=$ Drosophilinae, Eug $=$ Euglossini, For $($ do $)=$ Formicidae de dosel, For $($ ho $)=$ Formicidae de hojarasca, For $=$ Formicidae, Het = Heterocera (Arctiidae, Saturniidae y Sphingidae), Ispo = Isopoda, Ispt $=$ Isoptera, $\mathrm{Ma}(\mathrm{gr})=$ Mamíferos grandes, $\mathrm{Ma}(\mathrm{pe})=$ Mamíferos pequeños, $\mathrm{Ma}=$ Mammalia, $\mathrm{Ne}=\mathrm{Nematoda}, \mathrm{Nym}$ = Nymphalidae, Oes = Oestroidea (Calliphoridae, Mesembrinellidae y Sarcophagidae), Pla = Plantas, Re = Reptilia, Rho = Rhopalocera "mariposas diurnas", Sca = Scarabaeinae, Vet = Vertebrados terrestres (Amphibia, Mammalia y Reptilia).

Abbreviations: $\mathrm{Am}=$ Amphibia, Ara = Araneae, Arac $=$ Arachnida (Amblypygi, Araneae, Opiliones, Scorpiones y Uropygi), $\mathrm{Chi}=$ Chiroptera $, \mathrm{Chil}=\mathrm{Chilopoda}, \mathrm{Col}(\mathrm{do})=$ Canopy beetles, $\mathrm{Col}(\mathrm{vo})=$ Flying beetles, $\mathrm{Col}=\mathrm{Coleoptera}$, Coll = Collembola, Dro = Drosophilinae, Eug = Euglossini, For (do) = Canopy ants, For (ho) = Leaflitter ants, For = Formicidae, Het $=$ Heterocera (Arctiidae, Saturniidae y Sphingidae), Ispo = Isopoda, Ispt $=$ Isoptera, Ma $(\mathrm{gr})=\mathrm{Big}$ mammals, $\mathrm{Ma}$ (pe) = Small mammals, $\mathrm{Ma}=$ Mammalia, $\mathrm{Ne}=$ Nematoda, $\mathrm{Nym}=$ Nymphalidae, Oes $=$ Oestroidea (Calliphoridae, Mesembrinellidae y Sarcophagidae), Pla = Plants, Re = Reptilia, Rho = Rhopalocera "diurnal butterflies", Sca $=$ Scarabaeinae, Vet $=$ Terrestrial vertebrates (Amphibia, Mammalia y Reptilia). 
abundancia, riqueza, composición y grupos funcionales observados con anterioridad en un muestreo exhaustivo.

Otro modo de ganar costo-efectividad en las evaluaciones de artrópodos terrestres es aprovechar un método de captura supuestamente dirigido o específico para colectardosomástaxones deartrópodos. Lovell et al. (2007) evaluaron de manera simultánea nueve taxones de invertebrados por medio de las siguientes combinaciones de métodos de captura: búsqueda directa por unidad de área para Blattodea, Chilopoda, Diplopoda y Gastropoda, vareo de arbustos para Araneae, Diplopoda y Gastropoda, censos visuales para Apoidea, Orthoptera y Rhopalocera, bandejas coloreadas para Apoidea y Araneae, y trampas cebadas con fruta fermentada para Apoidea, Cetoniinae y Rhopalocera. Cardoso et al. (2008) comprobaron que un protocolo de colecta dirigido a inventariar la riqueza de arañas (Araneae) en un bosque de robles del Mediterráneo puede ser expandido hacia una mayor variedad de artrópodos sin comprometer la capacidad de los colectores ni demandar mayores recursos. Uehara-Prado et al. (2009) utilizaron trampas cebadas con plátano fermentado para evaluar comunidades de mariposas diurnas y observaron que además de éstas fueron atraídos muchos ejemplares del género Matusinhosa (Coleoptera, Oedemeridae). Las especies de Matusinhosa colectadas de esta manera, evidenciaron una marcada preferencia por las localidades de bosque no perturbado.

\section{CONSIDERACIONES FINALES}

Los cuatro argumentos presentados plantean posibilidades interesantes para incluir a los artrópodos en evaluaciones ambientales y abarcan tanto las ventajas metodológicas como también la información ecológica que podría obtenerse al evaluarlos. Sin embargo, la transmisión e implementación de estos argumentos requiere el convencimiento de distintos actores sociales. Uno de los medios para conseguirlo es disponer de categorías de conservación de especies que sean reconocidas por la legislación gubernamental, tal y como ha ocurrido con los vertebrados. En ese aspecto, los artrópodos presentan un gran déficit puesto que no es sencillo precisar la distribución geográfica, endemismo, rareza y vulnerabilidad para centenas, miles o incluso decenas de miles de especies. Los progresos más notables hacia dicho objetivo han sido obtenidos para las mariposas diurnas (Papilionoidea), al punto que la información disponible ha permitido establecer tendencias globales (Lewis \& Senior 2011). Otro grupo que ha recibido creciente atención son los coleópteros saproxílicos en el Norte de Europa; para ellos se han implementado monitoreos a largo plazo (Martikainen \& Kaila 2004) y se han propuesto subconjuntos de especies que sirven como indicadores de su biodiversidad (Sebek et al. 2012).

\section{BIBLIOGRAFÍA}

ANDERSEN, A. 1999. My bioindicator or yours? Making the selection. Journal of Insect Conservation, 3:61-64.

ANDERSEn, A; BD HofFMAnN; WJ Muller \& AD GRIfFiths. 2002. Using ants as bioindicators in land management: simplifying assessment of ant community responses. Journal of Applied Ecology, 39:8-17.

BANGert, RK \& CN SLOBOdChiKoff. 2006. Conservation of prairie dog ecosystem engineering may support arthropod beta and gamma diversity. Journal of Arid Environments, 67:100-115.

Bisevac, L \& J Majer. 2002. Cost effectiveness and datayield of biodiversity surveys. Journal of the Royal Society of Western Australia, 85:129-132.

Bolger, DT; AV SuARez; KR CROOKS; SA Morrison \& TJ CASE. 2000. Arthropods in urban habitat fragments in southern California: area, age, and edge effects. Ecological Applications, 10:1230-1248.

CARdoso, P; PAV Borges \& C GASPAR. 2007. Biotic integrity of the arthropod communities in the natural forests of Azores. Biodiversity and Conservation, 16:2883-2901.

Cardoso, P; TL ERwin; PAV Borges \& TR New. 2011. The seven impediments in invertebrate conservation and how to overcome them. Biological Conservation, 144: 2647-2655.

Cardoso, P; N ScharfF; C Gaspar; SS Henrigues; R CarvalHo; Et Al. 2008. Rapid biodiversity assessment of spiders (Araneae) using semi-quantitative sampling: a case study in a Mediterranean forest. Insect Conservation and Diversity, 1:71-84.

CHAN, EKW; Y ZHANG \& D DudGEON. 2007. Contribution of adult aquatic insects to riparian prey availability along tropical forest streams. Marine and Freshwater Research, 58:725-732.

CHIN, K \& BD GILL. 1996. Dinosaurs, dung beetles and conifers: Participants in a Cretaceous food web. Palaios, 11:280-285.

FAbricius C; M Burger \& PAR Hockey. 2003. Comparing biodiversity between protected areas and adjacent rangeland in xeric succulent thicket, South Africa: arthropods and reptiles. Journal of Applied Ecology, 40: 392-403.

Ferreira, RL \& LCS Horta. 2001. Natural and human impacts on invertebrate communities in brazilian caves. Revista Brasileira de Biologia, 61(1):7-17.

Gardner TA; J Barlow; IS Araujo; TC Ávila-Pires; AB BONALDO; ET AL. 2008. The cost-effectiveness of biodiversity surveys in tropical forests. Ecology Letters, 11:139-150.

Gaston, KJ; SL ChOwn \& RD MerCer. 2001. The animal 
species-body size distribution of Marion Island. Proceedings of Natural Academy of Sciences, 98:1449314496.

Gratton, C \& RF DenNo. 2005. Restoration of arthropod assemblages in a Spartina salt marsh following memoval of the invasive plant Phragmites australis. Restoration Ecology, 13:358-372.

Harris, RJ; RJ Toft; JS Dugdale; PA Williams \& JS Rees. 2004. Insect assemblages in a native (kanuka - Kunzea ericoides) and an invasive (gorse - Ulex europaeus) shrubland. New Zealand Journal of Ecology, 28:35-47.

HASKELL, DG. 2000. Effects of forest roads on macroinvertebrate soil fauna of the Southern Appalachian Mountains. Conservation Biology, 14:5763.

HolLING, CS. 1992. Cross-scale morphology, geometry and dynamics of ecosystems. Ecological Monographs, 62:447-502.

KARR, JR \& DN KIMBERLING. 2003. A terrestrial arthropod index of biological integrity for shrub-steppe landscapes. Northwest Science, 77:202-213.

Labandeira, CC; DL Dilcher; DR Davis \& DL Wagner. 1994. Ninety-seven million years of angiosperm-insect association: Paleobiological insights into the meaning of coevolution. Proceedings of the Natural Academy of Sciences, 91:12278-12282.

Lawton, JH; DE Bignell; B Bolton; GF Bloemers; P EGGLETON; ET AL. 1998. Biodiversity inventories, indicator taxa and effects of habitat modification in tropical forests. Nature, 391:72-75.

LewIS, OT \& MJM SENIOR. 2011. Assessing conservation status and trends for the world's butterflies: the Sampled Red List Index approach. Journal of Insect Conservation, 15(1-2):121-128.

Losey, JE \& M Vaughan. 2006. The economic value of ecological services provided by insects. BioScience, 56(4):311-323.

Lovell, S; M Hamer; R Slotow \& D Herbert. 2007. Assessment of congruency across invertebrate taxa and taxonomic levels to identify potential surrogates. Biological Conservation, 139:113-125.

Maleque, MA; K Maeto \& HT Ishit. 2009. Arthropods as bioindicators of sustainable forest management, with a focus on plantation forests. Applied Entomology and Zoology, 44(1):1-11.

Margalef, R. 1991. Teoría de los Sistemas Ecológicos. Publicacions de la Universitat de Barcelona. Universitat de Barcelona. Barcelona (España). Pp. 290.

Martikainen, P \& L Kaila. 2004. Sampling saproxylic beetles: lessons from a 10-year monitoring study. Biological Conservation, 120:171-181.

Melic, A. 2003. De los jeroglíficos a los tebeos: Los artrópodos en la Cultura. Boletín de la Sociedad Entomológica Aragonesa, 32:325-357.

Nichols, E; TA Gardner; CA Peres; S Spector \& The Scarabaeinae Research Network. 2009. Co-declining mammals and dung beetles: an impending ecological cascade. Oikos, 118(4):481-487.

Noss, RF. 1990. Indicators for monitoring biodiversity: A hierarchical approach. Conservation Biology, 4(4): 355-364.

Palmer, M \& GX Pons. 1996. Diversity in Western Mediterranean islets: effects of rat presence on a beetle guild. Acta Oecologica, 17(4):297-305.

Paoletti, MG; LJ Thomson \& AA Hoffmann (eds.). 2007. Using invertebrate bioindicators to assess agricultural sustainability in Australia: proposals and current practices. Australian Journal of Experimental Agriculture, 47(4):379-478

RiberA, I \& G Foster. 1997. El uso de artrópodos como indicadores biológicos. Boletín de la Sociedad Entomológica Aragonesa, 20:265-276.

Sebek, P; T Barnouin; A Brin; H Brustel; M Dufrêne; et AL. 2012. A test for assessment of saproxylic beetle biodiversity using subsets of "monitoring species". Ecological Indicators, 20:304-315.

Smith, RM; PH Warren; K Thompson \& KJ Gaston. 2006. Urban domestic gardens (VI): environmental correlates of invertebrate species richness. Biodiversity and Conservation, 15:2415-2438.

ThOMAs, CFG \& EJP MARSHALl. 1999. Arthropod abundance and diversity in differently vegetated margins of arable fields. Agriculture, Ecosystems and Environment, 72:131-144.

Turnhout, E; M Hisschemöller \& H EiJSACKERs. 2007. Ecological indicators: between the two fires of science and policy. Ecological Indicators, 7:215-228.

Uehara-Prado, M; J de Oliveira Fernandes; A de Moura Bello; G Machado; AJ Santos; et al. 2009. Selecting terrestrial arthropods as indicators of small-scale disturbance: A first approach in the Brazilian Atlantic Forest. Biological Conservation, 142:1220-1228.

Vtorov, IP. 1993. Feral pig removal: effects on soil microarthropods in a Hawaiian rain forest. Journal of Wildlife Management, 57(4):875-880.

Wardwell, DA; CR Allen; CD Peterson \& A J Tyre. 2008. A test of the crossscale resilience model: Functional richness in Mediterranean-climate ecosystems. Ecological Complexity, 5:165-182. 